LIAMES 8 - pp. 105-120, Primavera 2008

Mara Santos ${ }^{1}$

(Universidade Federal do Rio de Janeiro - UFRJ)

\title{
As classes morfológicas flexionais da língua kuikuro
}

\begin{abstract}
This article aims to discuss the morphological inflectional classes of the Kuikuro language, one of the variants of the Upper Xingu Karib languages, which comprise the southern branch of the Karib family (Franchetto and Meira, 2005). The inflectional morphological classes organize the language in classes that are explicitly ordered and that go through several productive inflectional and derivative processes. The Kuikuro language has a rich set of functional morphemes responsible for aspectual inflection, and Aspect is very important to the formation of the verb in the unmarked descriptive mood. The allomorphs of the Mood Aspects (Descriptive, Punctual, Continuative and Perfective) are distributed in five inflectional classes. We show that there is no phonological motivation for explaining the distribution of these allomorphs. The same can be observed on the so-called participle forms. The division of the Aspects into five inflectional morphological classes results from the analysis of both the function and distribution of these allomorphs that are affixed on verbs. We propose that this occurs because of an arbitrary property of the root that is a formal property without semantic or, as mentioned above, a phonological motivation.
\end{abstract}

KEYWORDS: Karib; Kuikuro; Verbal classes; Nominalization; Verbalization.

\section{RESUMO}

Este artigo tem como foco a organização das classes morfológicas flexionais da língua kuikuro, uma das variantes da língua karib alto-xinguana, que constitui um dos ramos meridionais da família lingüística karib (Franchetto e Meira, 2005). As classes morfológicas organizam a língua kuikuro em conjuntos explicitamente ordenados, que atravessam diversos processos flexionais e derivacionais. A língua kuikuro dispõe de um rico conjunto de morfemas funcionais responsáveis pela flexão aspectual, e o Aspecto é indispensável na formação da palavra verbal no que podemos chamar de Modo Descritivo, não marcado. Os alomorfes dos Aspectos do Modo Descritivo, Pontual, Continuativo e Perfeito se distribuem em cinco classes flexionais exclusivamente morfológicas, não existindo qualquer condicionamento fonológico que possa ser considerado responsável por tal alomorfia. O mesmo fenômeno pode ser observado nas formas que chamamos de "participiais". A organização dos Aspectos em cinco classes

${ }^{1}$ Doutora em lingüística pela UFRJ. Participa do projeto Documentação da Língua Carib do Alto Xingu, coordenado pela doutora Bruna Franchetto, com apoio da VolkswagenStiftung, CNPq e FINEP.

${ }^{2}$ Este trabalho é parte do capítulo 3 da Tese de Doutorado, defendida na Pós-Graduação de Lingüística da Universidade Federal do Rio de Janeiro e orientada pela doutora Bruna Franchetto, intitulada Morfologia kuikuro - Gerando nomes e verbos (Santos, 2007), <mara_fsantos@yahoo.com.br>. 
morfológicas flexionais é produto da observação do comportamento de cada alomorfe após os verbos aos quais ele se sufixa. Trata-se de uma propriedade arbitrária da raiz, uma propriedade puramente formal que não indica nenhuma motivação semântica, nem, tampouco, qualquer condicionamento fonológico.

PALAVRAS-CHAVE: Karib; Kuikuro; Classes verbais; Nominalização; Verbalização.

\section{INTRODUÇÃO}

Este artigo tem como foco a organização das classes morfológicas flexionais da língua kuikuro, uma das variantes da língua karib alto-xinguana, que constitui um dos ramos meridionais da família karib (Franchetto e Meira, 2005). A língua karib alto-xinguana é constituída por duas variantes dialetais: de um lado, a falada pelos Kalapalo, Matipú e Nahukwá, e, de outro, a falada pelos Kuikuro. Todos esses grupos habitam a região dos formadores orientais do rio Xingu, ao sul da terra indígena do Xingu, estado de Mato Grosso.

As classes morfológicas organizam a língua kuikuro em conjuntos explicitamente ordenados, que atravessam diversos processos flexionais e derivacionais. Este trabalho aborda a flexão verbal dos Aspectos do Modo Descritivo: Pontual, Continuativo e Perfeito. Estes apresentam uma rica alomorfia morfologicamente condicionada, não existindo qualquer condicionamento fonológico, que se organiza em cinco classes flexionais. $\mathrm{O}$ mesmo fenômeno pode ser observado nas formas que chamamos de "participiais", nas nominalizações de argumento externo (Agente) e de argumento interno (Paciente, Tema, Ator), na flexão nominal, ou seja, na alomorfia dos sufixos de posse ou de relação ou dependência.

O Kuikuro é uma língua de núcleo final e ergativa do ponto de vista da tipologia morfossintática (Franchetto, 1986): o argumento com papel temático de Agente é sempre marcado pela posposição $\{\boldsymbol{h e} \boldsymbol{e} \boldsymbol{e}\}$, seja pronome, seja nome pleno. Essa manifestação morfológica tem sido tradicionalmente chamada de marca de Caso Ergativo. O Agente de verbos transitivos é tratado diferentemente do Objeto e do argumento único dos verbos intransitivos, que não recebem nenhuma marca morfológica (Caso Absolutivo). Esse padrão se reitera também na ordem dos constituintes e no vínculo fonológico entre o verbo e os seus argumentos internos ( $\mathrm{S}$ (ujeito intransitivo) e $\mathrm{O}$ (bjeto transitivo)).

O propósito deste artigo é contribuir com análises empiricamente fundamentadas acerca do fenômeno das classes morfológicas que ocorre em Kuikuro. Na primeira parte do artigo, analisamos os processos de formação da palavra verbal, a estrutura interna, identificando morfemas lexicais e funcionais, como as raízes e seus categorizadores; na segunda parte, a organização das cinco classes morfológicas flexionais e na terceira parte as classes verbais e o "sistema $* t-V$-ce" das línguas karib.

\section{AFORMAÇÃO DAPALAVRA VERBAL}

A palavra verbal em Kuikuro apresenta a seguinte estrutura: 
(Pr)-(MO)-(DTR)-RAIZ-VBLZ(Tr/Int)-TR-(MOD)-ASP-(PL)-(T) ${ }^{3}$

Consideramos a palavra verbal em Kuikuro não como uma categoria pré-sintática associada a itens lexicais, mas como resultado de operações sintáticas, conforme a proposta da Morfologia Distribuída ${ }^{4}$. A palavra verbal assume sua função quando realizada em configurações sintáticas, uma vez que tenham sido concatenados dois primitivos sintáticos, raízes e categorizadores verbais. Assim, em Kuikuro, um radical verbal consistiria de $\sqrt{ } \mathrm{R} /$ $\mathrm{N}+$ morfema verbalizador, podendo o verbalizador ser fonologicamente nulo ou realizado explicitamente. Até agora identificamos 11 morfemas verbalizadores em Kuikuro: 5 formam verbos intransitivos e 6 formam verbos transitivos. Cada verbalizador possui traços formais, fonológicos e semânticos.

Represento nas estruturas que se seguem a fusão da raiz com a morfologia verbalizadora:

(i) verbos formados pelo processo de concatenação de um Nome com morfologia verbalizadora realizada explicitamente $\left[\mathrm{v}_{1}-\right.$ estativos (intransitivos); $\mathrm{v}_{2}$ - causativos (transitivos) $]^{5}:(\sqrt{ } \mathrm{N}+\mathrm{VBLZ})$.
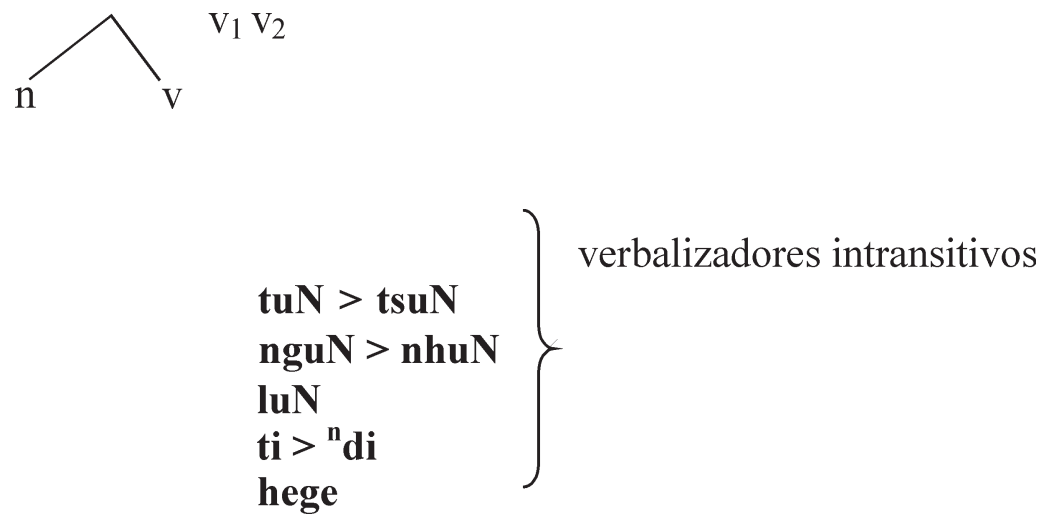

${ }^{3} \operatorname{Pr}$ (prefixo pronominal); MO (Marcador de Objeto); DTR (detransitivizador); VBLZ (verbalizador transitivo ou intransitivo); TR (transitivizador); MOD (modo); ASP (aspecto); PL (plural), T (tempo).

${ }^{4}$ Minha análise baseia-se no modelo da teoria Morfologia Distribuída (Halle e Marantz, 1993; Harley e Noyer, 1998, Embick e Halle, 2004). Neste artigo me limitarei apenas a descrever o processo de formação da palavra verbal sem aprofundar na base teórica que subsidiou a minha análise (Santos, 2007).

${ }^{5}$ As convenções da transcrição ortográfica usada neste artigo são também usadas pelos Karib do Alto Xingu em sua escrita: $\mathrm{i} \rightarrow \mathrm{u} ; \mathrm{j} \rightarrow \mathrm{j}$; tepe uvular $\rightarrow \mathrm{g} ; \mathrm{y} \rightarrow \mathrm{ng} ; \mathrm{n} \rightarrow \mathrm{nh} ;{ }^{\mathrm{n}} \mathrm{g} \rightarrow \mathrm{nkg}$. 


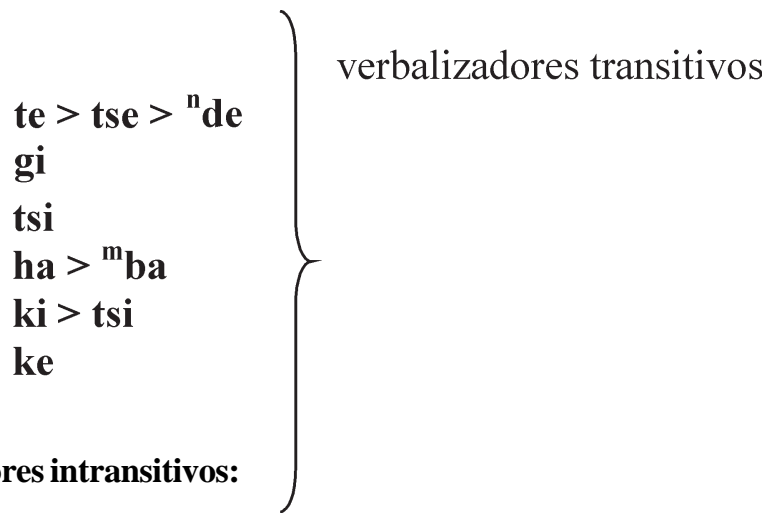

morfemas verbalizadores intransitivos:

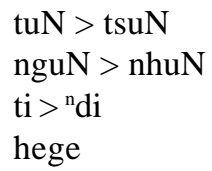

Os morfemas verbalizadores intransitivo tuN $>\mathbf{t s u N}$ produzem radicais verbais da classe 1 com semântica de "estar no estado de ter X", sendo X um Nome:

1a) ege (Nome) "preguiça"

1b) u-ege-sü

1-preguiça-REL

heke u-e-tagü

"a minha preguiça está-me matando (estou com muita preguiça)"

1c.) u-ege-tsuN-tagü > uegetsundagü (Verbo)

1-preguiça-VBLZ-CONT

"eu estou com preguiça"

\section{Morfemas verbalizadores transitivos:}

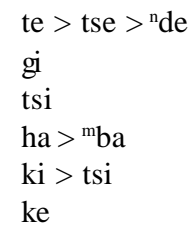

Os verbalizadores transitivos te $>$ tse $>$ " $\mathbf{n d e}$ formam verbos de classes diferentes e realizam de modo fonologicamente diferente uma mesma semântica: "causar (causa externa) $\mathrm{X}$ (beneficiário) ter (estar com) Y (tema)". Vejamos um exemplo com o verbalizador transitivo te que produz radicais verbais da classe 5 : 
2a) imbuta (Nome)

"remédio"

2b) u-imbuta-gü

1-remédio-REL

"meu remédio"

2c)

$\begin{array}{lll}u \text {-hisuü-gü } & \text { imbuta-te-lü } & \text { u-heke } \\ 1 \text { - irmão-REL } & \text { remédio-VBLZ) }\end{array}$

"eu dei remédio para meu irmão"

(ii) verbos formados por verbalizadores fonologicamente nulos $(\varnothing)(\sqrt{R}+\mathrm{VBLZ})$. Os verbalizadores fonologicamente nulos formam verbos da classe 2, 3, 4 e 5, com exceção dos verbos da classe 1 , que são formados por verbalizadores fonologicamente realizados:

$\left[[\sqrt{ } \mathrm{R}] \mathrm{v}^{0}\right]$

3)

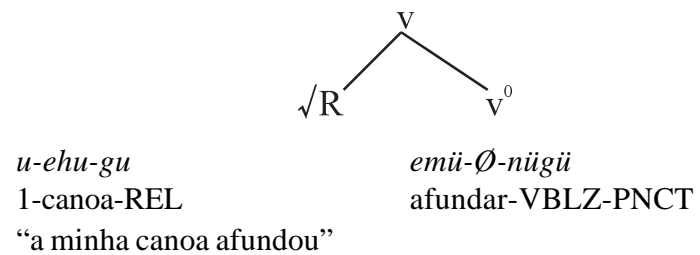

\section{AS CLASSES MORFOLÓGICAS FLEXIONAIS}

O Kuikuro dispõe de um rico conjunto de morfemas funcionais responsáveis pela flexão aspectual, e o Aspecto é indispensável na formação da palavra verbal no que podemos chamar de Modo Descritivo, não marcado. Não há, no verbo, uma flexão propriamente de Tempo (Tense), com exceção do sufixo -ingo. Esse morfema fecha a palavra, podendo ser seguido apenas pela cópula -i. O sufixo -ingo pode ocorrer tanto com verbos como com nomes, não se submetendo à lógica das classes morfológicas.

Franchetto $(1986,2005,2006)$ afirma que, para o Modo Descritivo, existem somente três possíveis Aspectos como flexão verbal:

- O Aspecto Pontual (PNCT), com sua alomorfia — V-lü ( $\boldsymbol{\varnothing}$, -nügü, -kügü, -jüi) —, é usado para descrever uma ação ou evento como fato instantâneo ou como um ponto sem nenhuma extensão temporal inerente, podendo ser interpretado como presente, passado ou futuro, ou seja, concomitante ou anterior ao evento de fala. Os próprios Kuikuro dizem que os verbos flexionados com esse Aspecto são "quase nome, quase coisa".

- O Aspecto Continuativo (CONT), com sua alomorfia — V-tagü (-tsagü, -gagü) —, é usado para descrever uma ação ou evento como processo, com extensão temporal inerente, no presente, no passado ou no futuro. 
- O Aspecto Perfeito (PERF), com sua alomorfia - V-pügü (-hügü, -tühügü e -tsühügü) -, denota uma ação ou evento que se completou (passado) e que produziu um estado definitivo ou resultativo.

A organização dos Aspectos em cinco classes (1, 2, 3, 4 e 5) é produto da observação do comportamento de cada alomorfe após os verbos aos quais ele se sufixa. Trata-se de uma propriedade arbitrária da raiz, uma propriedade puramente formal que não indica nenhuma motivação semântica, nem, tampouco, como já disse, qualquer condicionamento fonológico.

Apresentamos, no quadro abaixo, alguns radicais verbais organizados nas cinco classes:

Quadro 1: Classes morfológicas flexionais

\begin{tabular}{|c|c|c|c|c|c|c|}
\hline Classe & 1 & \multicolumn{2}{|c|}{2} & 3 & 4 & 5 \\
\hline $\begin{array}{l}\text { cont. } \\
\text { pont. } \\
\text { perfc. }\end{array}$ & $\begin{array}{l}\text {-tagü } \\
\emptyset \\
\text {-hügü }\end{array}$ & \multicolumn{2}{|c|}{$\begin{array}{c}\text {-tagü } \\
\text {-nügü> kügü } \\
\text {-tühügü }\end{array}$} & $\begin{array}{l}\text {-tagü } \\
\text {-lü } \\
\text {-pügü }\end{array}$ & $\begin{array}{c}\text {-tsagü } \\
\text {-jü } \\
\text {-tsühügü }\end{array}$ & $\begin{array}{l}\text {-gagü } \\
\text {-lü } \\
\text {-pügü }\end{array}$ \\
\hline $\begin{array}{l}\text { Verbalizador } \\
\text { transitivo }\end{array}$ & & \multicolumn{2}{|c|}{$\varnothing$} & $\begin{array}{c}\emptyset, \text { tsi }>\text { ki, } \\
\text { te }>\text { nde, } \\
\text { ha }>\text {-mba }\end{array}$ & $\varnothing$, tsi & $\varnothing$, te \\
\hline $\begin{array}{c}\text { Verbo } \\
\text { transitivo }\end{array}$ & & $\begin{array}{l}\text { ongindagü } \\
\text { (ongiN-Ø-tagü) } \\
\text { enterrar } \\
\text { tundagü } \\
\text { (tuN-Ø-tagü) } \\
\text { dar }\end{array}$ & $\begin{array}{l}\text { ane-Ø-tagü } \\
\text { queimar } \\
\text { anhe-Ø-tagü } \\
\text { perder }\end{array}$ & $\begin{array}{l}\text { agi-Ø-tagü } \\
\text { jogar } \\
\text { he-Ø-tagü } \\
\text { quebrar } \\
\text { iküpi-tsi- } \\
\text { tagü } \\
\text { tirar barba } \\
\text { ãü-ki-tagü } \\
\text { tirar piolho }\end{array}$ & $\begin{array}{l}\text { agugi-Ø- } \\
\text { tsagü } \\
\text { rachar } \\
\text { ahükügi- } \\
\text { Ø-tsagü } \\
\text { diminuir } \\
\text { ege-tsi-jü } \\
\text { sentir } \\
\text { preguiça }\end{array}$ & $\begin{array}{l}\text { api-Ø-gagü } \\
\text { bater } \\
\text { hote-Ø- } \\
\text { gagü } \\
\text { queimar } \\
\text { imbuta-te- } \\
\text { lü } \\
\text { dar remédio }\end{array}$ \\
\hline $\begin{array}{c}\text { Verbo } \\
\text { detransitivizado }\end{array}$ & & $\begin{array}{l}\text { otonginügü } \\
\text { (ot-ongiN-Ø- } \\
\text { nügü) } \\
\text { enterrar-se }\end{array}$ & $\begin{array}{l}\text { at-ane-Ø- } \\
\text { nügü } \\
\text { queimar-se }\end{array}$ & $\begin{array}{l}\text { at-agi-Ø- } \\
\text { tagü } \\
\text { jogar-se }\end{array}$ & $\begin{array}{l}\text { at-agugi- } \\
\varnothing \text {-jü } \\
\text { rachar-se }\end{array}$ & $\begin{array}{l}\text { ag-api-Ø-lü } \\
\text { bater-se }\end{array}$ \\
\hline $\begin{array}{l}\text { Verbalizador } \\
\text { intransitivo }\end{array}$ & $\begin{array}{l}\text { tuN }>\text { tsuN, } \\
\text { nguN }>\text { nhuN }\end{array}$ & \multicolumn{2}{|c|}{$\varnothing$} & $\emptyset, \mathrm{ti}>$ ndi & $\varnothing$ & $\varnothing$, hege \\
\hline $\begin{array}{c}\text { Verbo } \\
\text { intransitivo }\end{array}$ & $\begin{array}{l}\text { angundagü } \\
\text { (a-nguN-tagü) } \\
\text { dançar } \\
\text { apüngundagü } \\
\text { (apü-nguN- } \\
\text { tagü) } \\
\text { morrer } \\
\text { ajotundagü } \\
\text { (ajo-tuN-tagü) } \\
\text { namorar }\end{array}$ & $\begin{array}{l}\text { akandagü } \\
\text { (akaN-Ø-tagü) } \\
\text { sentar } \\
\text { endagü } \\
\text { (eN-Ø-tagü) } \\
\text { entrar }\end{array}$ & $\begin{array}{l}\text { ije-Ø-tagü } \\
\text { nadar } \\
\text { ale-Ø-tagü } \\
\text { encher }\end{array}$ & $\begin{array}{l}\text { alahi-Ø tagü } \\
\text { abaixar } \\
\text { umutundilü } \\
\text { (umutuN-ti- } \\
\text { lï) } \\
\text { florescer } \\
\text { amatso-ti- } \\
\text { tagü } \\
\text { menstruar }\end{array}$ & $\begin{array}{l}\text { ahu-Ø- } \\
\text { tsagü } \\
\text { encher } \\
\text { aku-Ø- } \\
\text { tsagü } \\
\text { estar de } \\
\text { barriga } \\
\text { cheia }\end{array}$ & $\begin{array}{l}\text { ihati-Ø- } \\
\text { gagü } \\
\text { sair } \\
\text { apü-Ø-gagü } \\
\text { amadurecer }\end{array}$ \\
\hline $\begin{array}{c}\text { Verbo } \\
\text { transitivizado }\end{array}$ & & & $\begin{array}{l}\text { a-ngu-ne- } \\
\text { nügü } \\
\text { fazer dançar }\end{array}$ & $\begin{array}{l}\text { alahi-Ø-ne- } \\
\text { tagü } \\
\text { fazer abaixar }\end{array}$ & $\begin{array}{l}\text { ukinhulu- } \\
\emptyset \text {-ki-tsagü } \\
\text { fazer, ter } \\
\text { ciúmes }\end{array}$ & \\
\hline
\end{tabular}


A partir do Quadro 1, é possível formular algumas generalizações a respeito do comportamento de cada classe.

(i) À classe 1 (CL1) pertencem verbos monoargumentais (intransitivos). Nessa classe, os verbos são formados pelos verbalizadores nguN, tuN, tsuN, guN, luN, com traços sintáticos de [-AG, -EXT] e traços semânticos de [ESTADO], concatenados a Nomes. Esses verbalizadores, como as raízes da classe 2 , se caracterizam, em sua representação fonológica subjacente, por ter um traço [nasal], assinalado aqui por [N]. Representamos esse tipo de raízes com o rótulo $\sqrt{ } \mathrm{RN}^{6}$. O traço [N] se realiza pré-nasalizando e vozeando a consoante oclusiva inicial dos sufixos (de Aspecto Continuativo, de Modo, nominalizadores, verbalizadores, de pluralização, entre outros). Disso decorre a alomorfia do Aspecto Continuativo -tagü >-ndagü. O Aspecto Pontual na classe 1 é ø; por restrição da estrutura silábica, o traço [N] não se realiza em posição final de sílaba. Veja-se o paradigma abaixo:

4)

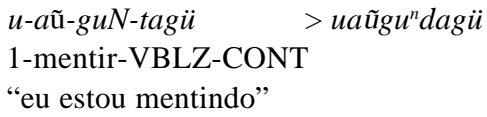

(ii) Na classe 2 (CL2) todos os verbos são formados por verbalizadores nulos (Ø). Os Aspectos Continuativo, -tagü, Pontual, -nügü, diferem dos da classe 1 por se acrescentarem a verbos tanto monoargumentais (intransitivos) como biargumentais (transitivos). Além disso, os verbos da classe 2 incluem o tipo $\sqrt{ } \mathrm{RN}$ [descrito em (i)] e verbos que não contêm [N]. Os verbos $\sqrt{ } \mathrm{RN}$, portanto, podem ter sufixos da classe 1 e da classe 2 , e, nesta última, eles podem ser tanto transitivos como intransitivos. Não detectamos nenhuma propriedade semântica comum aos intransitivos $\sqrt{ } \mathrm{RN}$ da classe 2 . Não existe qualquer evidência que indique uma diferença de sentido entre o Pontual -nügü e o Pontual $\emptyset$ ou uma diferença de sentido entre verbos intransitivos $\sqrt{ } \mathrm{RN}$ com -nügü e $\boldsymbol{\emptyset}$. O sentido do Aspecto Pontual se mantém constante: o de um evento visto como ponto ou instante sem extensão temporal (um não-processo):

6)

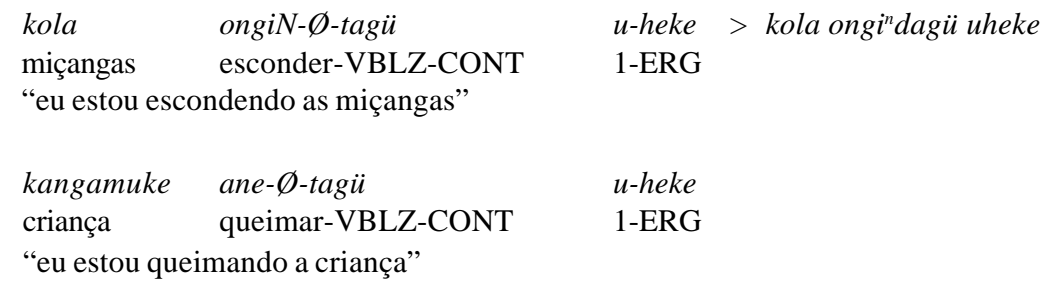

${ }^{6}$ Devemos a Sérgio Meira a sugestão de analisar a nasalidade em questão como traço da raiz ou do verbalizador. 
"a minha canoa afundou"

Na classe 2 temos uma alternância entre as formas do Aspecto Pontual -nügü kügü, equivalentes segundo nossos consultores kuikuro, embora -nügü seja mais freqüente:

$$
\text { u-ikeni-Ø-kügü nika e-heke }
$$

1-acreditar-VBLZ-PNCT Q 2-ERG

"você acredita em mim?"

10)

akinha ikeni- Ø-nügü u-heke

estória acreditar-VBLZ-PNCT 1-ERG

"eu acreditei na estória"

11) konige u-hanga-nkgi-kügü u-enkgu-püa gele u-etene-gü heke ontem 1-orelha-VBLZ-PNCT 1-porto-exLOC ainda 1-remo-REL ERG "ontem esqueci o meu remo onde eu aportei"

12) ihitsão heke leha inh-undi- Ø-kügü

mulheres ERG CMPL MO-zombar-VBLZ-PNCT

"eram as mulheres que tinham zombado dele"

(iii) Na classe 3 (CL3) temos verbos formados pelos verbalizadores $\emptyset$, tsi, te, $\mathbf{k i}$, ti, intransitivos e transitivos. Nessa classe, as formas aspectuais são: Continuativo -tagü, Pontual -lü, Perfectivo -pügü:

13)

$\begin{array}{lll}\text { api } & \text { aka- } \emptyset \text {-ne-tagü } & \text { u-heke } \\ \text { avô } & \text { sentar-VBLZ-TR-CONT } & 1 \text {-ERG }\end{array}$

14)

ngongo gekunu heke u-itongoN-ki-lü

terra poeira ERG 1-tosse-VBLZ-TR-PNCT

"a poeira me fez tossir"

15)

$\begin{array}{lll}\text { kanga } & \text { he- } \emptyset \text {-pügü } & \text { leha } \\ \text { peixe matar-VBLZ-PERF } & \text { CMPL } \\ \text { "o peixe já está morto" } & \end{array}$

(iv) Na classe 4 (CL4), como na classe 3, temos verbos mono e biargumentais, formados, agora, pelos verbalizadores $\emptyset$ e tsi, com o Aspecto Continuativo -tsagü e o Aspecto Pontual -jü. Poderíamos pensar em condicionamento fonológico, mas ele não é consistente. As mudanças $\mathbf{t}>\mathbf{t} \mathbf{e}$ e $\mathbf{l}>\mathbf{j}$, resultado de coronalização (palatalização) após vogal coronal ([i]), são processos regulares em Kuikuro, em fronteira morfológica (Franchetto, 1995). O processo de "coronalização", todavia, não funciona exaustivamente na alomorfia dos 
sufixos aspectuais Continuativo, -tagü/-tsagü, e Pontual, -lüi/-jü. A grande maioria das raízes possui, de fato, a vogal [i] em posição final, mas encontramos não poucas exceções, como inaü-tsagü, "apertar"; inu-jü, "entortar/dobrar"; itü-jü, "responder"; hu-tsagü, “inchar”. Se houve condicionamento fonológico, ele não é mais operante:

16)

$\begin{array}{ll}\text { pape ahehi- } \emptyset \text {-tsagü } & \text { u-heke } \\ \text { carta escrever-VBLZ-CONT } & 1 \text {-ERG } \\ \text { "eu estou escrevendo uma carta" } & \end{array}$

17) $\quad i \quad$ at-agugi- $\varnothing$-jü

árvore 3DTR-rachar-VBLZ-PNCT

"a árvore rachou-se"

18)

$\begin{array}{lll}\text { u-ingunkgi-ngu-ki-jü } & \text { akinha-gü } & \text { heke } \\ \text { 1-olho-VBLZ-TR-PNCT } & \text { história-REL } & \text { ERG } \\ \text { "a sua estória me fez pensar" } & & \end{array}$

(v) À CL5 pertencem verbos transitivos, intransitivos e detransitivizados formados pelos vebalizadores $\emptyset$ e tsi:

$19)$

$\begin{array}{ll}\text { katsogo api- } \emptyset \text {-lü } & \text { i-heke } \\ \text { cachorro bater-VBLZ-PNCT } & 3 \text {-ERG } \\ \text { "ele bate no cachorro" } & \end{array}$

"ele bateu no cachorro"

20)
u-g-api- Ø-gagü
martelu-ki
1-DTR-bater-VBLZ-CONT
martelo-INST
"eu me bati com martelo"

Como mais uma evidência a favor da existência de classes morfológicas flexionais em Kuikuro, a análise das raízes homófonas nos auxilia na comprovação de que estamos diante de um condicionamento não-fonológico, mas, sim, morfológico. As diferentes formas fonológicas dos morfemas de Aspecto são sufixadas a radicais verbais com sintaxe e semântica distintas.

Observem os exemplos abaixo ( Vt = verbo transitivo ou com Causa Externa, $\mathrm{Vi}=$ verbo intransitivo ou monoargumental ou com Causa Interna; Franchetto e Santos, 2003, Franchetto, 2006):

(i) Vt-CL3 (ASPC-CONT-tagü, PNCT-lü)

$\begin{array}{llll}\text { 21) ana } & \text { ahu- } \emptyset \text {-tagü } & \text { itão } & \text { heke } \\ \text { milho } & \text { pilar-VBLZ-CONT } & \text { mulher } & \text { ERG } \\ \text { "a mulher está pilando milho" } & & \end{array}$


Vi-CL4 (ASPC-CONT-tsagü)

22) tunga ahu- Ø-tsagü

rio encher-VBLZ-CONT

"o rio está enchendo"

(iii) Vi-CL3 (ASPC - CONT -tagü)

23) ana ati-Ø-tagü

milho brotar-VBLZ-CONT

"o milho está brotando"

(iv) Vt-CL4 (ASPC-CONT-tsagü)

24) ahukugu ati-Ø-tsagü u-heke

panela limpar-VBLZ-CONT 1-ERG

"eu estou limpando a panela"

Os dados acima mostram com clareza que não há condicionamento fonológico dos alomorfes de Aspecto Continuativo (-tagü, -tsagü) e que o fenômeno é de classes morfologicamente condicionadas.

Sabemos que homofonia e classes flexionais morfológicas são os resultados de processos diacrônicos como queda de vogais instáveis, redução silábica e assimilação (espraiamento dos traços coronal, labial, harmonia vocálica, fenômenos ainda produtivos em Kuikuro em outros contextos). A questão é, aqui, entender os fatos sincrônicos, em que processos morfofonológicos não são mais ativos?

\section{AS CLASSES VERBAIS E O SISTEMA *t-V-ce DAS LÍNGUAS KARIB}

Gildea (1998:218 e segs.), ao reconstruir a protomorfossintaxe karib, descreve o sistema *t-V-ce como uma das possíveis origens das construções ergativas nas línguas da família, graças a uma cadeia de gramaticalização pela qual, através de uma seqüência de etapas de reanálise, uma construção participial, acompanhada por auxiliar/cópula, é reanalisada como uma passiva (com auxiliar/cópula e agente marcado não-obrigatório) e, depois, como uma construção ergativa (sem auxiliar e com agente obrigatoriamente marcado). Em algumas línguas karib, a forma participial é sempre seguida pelo nominalizador *-mi, do qual deriva o nominalizador kuikuro -nhü. $\mathrm{O}$ autor assim descreve a protoforma *t-V-ce: *t(i)- "Adverbial"; *-ce "Participial" com seus alomorfes -se, -so, -ze, - -se, -tze, -

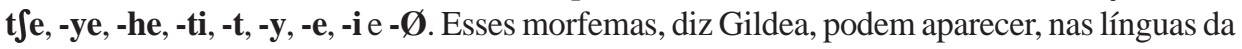
família, separadamente ou combinados um com outro.

${ }^{7}$ Ver Santos (2005). Raízes homófonas em Kuikuro. XVII Congresso da ABRALIN — Brasília, 17-19 de fevereiro de 2005. 
Como nas outras línguas karib, em Kuikuro esse sistema é marcado com o sufixo de flexão aspectual de particípio e a posição argumental é preenchida por uma única forma prefixal, glosada por nós como terceira pessoa anafórica $\mathbf{t}(\mathrm{t} \mathbf{i})$-. Os verbos resultantes desse sistema têm formas verbais Participiais, algo concluso. A língua kuikuro "escolheu" algumas das formas karib, organizando-as pela lógica de classes morfológicas: tü-/ tVerbo -i/-ti/-si/-acento. Elas se afixam a um radical verbal que pode ser transitivo, transitivizado, intransitivo ou detransitivizado. Observem a sua distribuição:

Quadro 2: As classes morfológicas do Particípio

\begin{tabular}{|c|c|c|c|c|c|c|}
\hline & CL1 & \multicolumn{2}{|c|}{ CL2 } & CL3 & CL4 & CL5 \\
\hline \multirow{6}{*}{ PTP } & $\begin{array}{l}\boldsymbol{t} \text { - aũg- } \boldsymbol{i} \\
\text { mentir }\end{array}$ & $\begin{array}{l}\text { tü-aka-ndi } \\
\text { sentar }\end{array}$ & $\begin{array}{l}t \text {-ije- } t \boldsymbol{i} \\
\text { nadar }\end{array}$ & $\begin{array}{l}t \text {-alahi } i \\
\text { abaixar }\end{array}$ & $\begin{array}{l}t \text {-ogopi-si } \\
\text { voltar }\end{array}$ & $\begin{array}{l}t \text {-ihat } i^{\prime} \\
\text { sair }\end{array}$ \\
\hline & $\begin{array}{l}\text { tü-het- } i \\
\text { gritar }\end{array}$ & $\begin{array}{l}\text { tü-e-ndi } \\
\text { entrar }\end{array}$ & $\begin{array}{l}t \text {-ale-t } \boldsymbol{t} \\
\text { encher }\end{array}$ & $\begin{array}{l}t \text {-apitsí } \\
\text { escorregar }\end{array}$ & $\begin{array}{l}t \text {-ili-si } \\
\text { beber }\end{array}$ & $\begin{array}{l}\boldsymbol{t} \text {-apü } \\
\text { amadurecer }\end{array}$ \\
\hline & \multirow[t]{4}{*}{$\begin{array}{l}t \ddot{u} \text {-hosig- } i \\
\text { sorrir }\end{array}$} & $\begin{array}{l}t \boldsymbol{t} \text {-emü-ndi } \\
\text { afundar }\end{array}$ & $\begin{array}{l}t \text {-anhe- } \boldsymbol{t} i \\
\text { perder }\end{array}$ & $\begin{array}{l}\text { tü̈-hé } \\
\text { matar }\end{array}$ & $\begin{array}{l}t \text {-agugi-si } \\
\text { rachar }\end{array}$ & $\begin{array}{l}\boldsymbol{t} \text {-atí } \\
\text { brotar }\end{array}$ \\
\hline & & \multirow[t]{3}{*}{$\begin{array}{l}\text { tun-di } \\
\text { dar }\end{array}$} & \multirow[t]{3}{*}{$\begin{array}{l}t \text {-akule-ne- } t \boldsymbol{i} \\
\text { fazer coar }\end{array}$} & \multirow[t]{3}{*}{$\begin{array}{l}\text { tü-hotsí } \\
\text { furar }\end{array}$} & $\begin{array}{l}\boldsymbol{t} \text {-ingunkgingu-ki-si } \boldsymbol{i} \\
\text { fazer pensar }\end{array}$ & \\
\hline & & & & & $\begin{array}{l}t \text {-ahu-si } \\
\text { alagado }\end{array}$ & \\
\hline & & & & & $\begin{array}{l}\boldsymbol{t} \text {-aku-si } \\
\text { estar de barriga } \\
\text { cheia }\end{array}$ & \\
\hline
\end{tabular}

O sentido da forma participial — glosada PTP — é de algo concluído, o que a aproxima do Aspecto Perfectivo, expresso pelo sufixo -pügü (e seus alomorfes), mas a isso acrescenta a idéia de um resultado que é condição, estado, propriedade do referente coindexado com o prefixo anafórico t(ï)-, que ocupa a posição de argumento absolutivo:

$\begin{array}{llll}t_{i} \text {-apü-ng- } i & \text { leha } & \text { ekege }_{i} & \text { leha } \\ \text { AN-maduro-VBLZ-PTP } & \text { CMPL } & \text { onça } & \text { CMPL }\end{array}$

"já morreu/tendo já morrido/já morta, a onça" 


$\begin{array}{llll}\text { ekege }_{i} & \text { leha } & t_{i} \text {-apü-ng-i } & \text { leha } \\ \text { onça } & \text { CMPL } & \text { AN-maduro-VBLZ-PTP } & \text { CMPL }\end{array}$

"a onça, já morreu/tendo já morrido/já morta"

t-apü-ng-i, "morto", é o estado resultante de um evento "morrer". Veja-se que o SN coindexado (ekege) está "livre" de sua relação de argumento absolutivo do verbo: ele pode ocorrer antes ou depois do verbo em forma participial, separado dele pela partícula leha, mantendo sua autonomia fonológica, já que não constitui uma unidade (sintática e fonológica) com o verbo, em outras palavras, não está na posição de argumento interno (absolutivo) do verbo ${ }^{8}$.

Apresentamos, a seguir, para cada classe, exemplos de radicais verbais nas formas participiais.

- Verbo intransitivo da $\mathrm{CL1}^{9}$ : o PTP se realiza com a forma: $t i i-/ t$ - Verbo + -i:

26)

$\begin{array}{llll}\text { ekege } & t \text {-apü-ngu-i } & \text { leha } & >t \text {-apünguN- } i>\text { tapüngi } \\ \text { onça } & \text { AN-maduro-VBLZ-PTP } & \text { CMPL } & \\ \text { "a onça, ela já está morta" } & \end{array}$

- CL2: o PTP se realiza com a forma: $t \ddot{i}-/ t-$ + Verbo + -ti:

27) akandoho leha t-ets-uhe- $\emptyset$ - $t i$

banco CMPL AN-2/3DTR-quebrar-VBLZ-PTP

"o banco, ele já está quebrado"

28) ahütü gele embuta tuN-ti-la u-heke inha

NEG ainda remédio AN dar-VBLZ-PTP-NEG 1-ERG 3-BEN

"ainda não foi dado o remédio para ele por mim"

- CL3: forma do Particípio Passado: tï-/t- + Verbo + acento na última sílaba do radical verbal:

29) ai leha t-amé u-heke

CMPL AN-gravar-PTP 1-ERG

"eu já o gravei"

30) lepe tü-hagakí i-heke, agi-Ø-lü leha i-heke

depois AN-arrancar-PTP 3-ERG jogar-VBLZ-PNCT CMPL 3-ERG

"depois dele tê-lo arrancado, ele o jogou fora"

${ }^{8}$ Para uma análise mais completa do sistema *t-V-ce, ver Santos (2007).

${ }^{9}$ Lembramos que na Classe1 há somente verbos intransitivos. 


\section{- CL4: forma do Particípio Passado: tï-/t- + Verbo + -si:}

31)

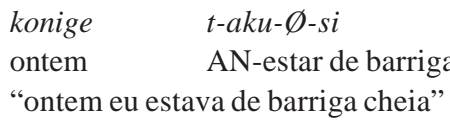

32)

$\begin{array}{llll}\text { u-alato-gu-pe } & \text { tsekegü-pe } & \text { t-at-agugi- } \emptyset \text {-si } & \text { leha } \\ \text { 1-tacho-REL-ex } & \text { grande-ex } & \text { AN-2/3DTR-rachar-VBLZ-PTP } & \text { CMPL } \\ \text { "o que foi meu tacho, o que era grande, ficou rachado" } & \end{array}$

- CL5: forma do Particípio Passado: tï-/t- + Verbo + acento na última sílaba do radical verbal, ou seja, a mesma forma vista para os verbos da CL3:
33) $\quad i$
tü-hoté leha árvore AN-queimar-PTP CMPL
"a árvore, ela já está queimada"

Seguem, agora, as formas participiais que ocorrem com verbos transitivizados pelos morfemas ne e ki:

(i) Com o transitivizador ne:

34) itão leha t-a-ngu-ne-ti mulher CMPL AN-dançar-VBLZ-TR-PTP

"a menina, foi feita dançar" (quando a menina sai pela primeira vez da reclusão pubertária para dançar em público)

(ii) Com o transitivizador ki:

36) u-ajo leha t-ingunkgi-ngu-ki-si u-heke

1-namorado CMPL AN-pensar-VBLZ-TR-PTP 1-ERG

"eu já pensei no meu namorado/o meu namorado, já foi pensado por mim"

$\begin{array}{llcc}u \text {-muku-gu } & \text { t-ipügelu- } \varnothing \text {-ki-si } & \text { kanga } & \text { heke } \\ \text { 1-filho-REL } & \text { AN-tremer-VBLZ-TR-PTP } & \text { peixe } & \text { ERG } \\ \text { "meu filho, foi feito tremer pelo peixe" (deu febre, fez mal) } & \end{array}$

É evidente que o sistema *t-V-ce manifesta significados e comportamentos sintáticos diferentes nas diversas línguas karib. Como vimos, em Kuikuro ele assume feições particulares, afastando-se dos padrões encontrados nas outras línguas. Os itens de vocabulário que realizam o Particípio apresentam quatro formas que se distribuem nas 
classes. A distribuição pelas classes não indica, como é de esperar, qualquer motivação fonológica ou semântica.

\section{CONSIDERAÇÕES FINAIS}

Como vimos, as classes morfológicas constituem, poderíamos dizer, uma linha de força estruturante que atravessa, se não toda, grande parte da morfossintaxe do Kuikuro. Aqui descrevemos como as classes morfológicas, explicitamente ordenadas, organizam os processos de flexão aspectual (Aspectos Pontual, Continuativo e Perfeito). Identificamos o condicionamento morfológico da alomorfia. Vimos como há uma distinção máxima de cinco classes morfológicas na flexão de Aspecto Pontual.

A descoberta da existência de cinco classes morfológicas flexionais para os verbos só foi possível após uma cuidadosa e ampla observação do comportamento de cada alomorfe dos sufixos de Aspecto do Modo Descritivo (Pontual, Continuativo e Perfectivo). Concluímos que não existe nenhum condicionamento semântico ou fonológico para tal fenômeno; a alomorfia dos sufixos de Aspecto (PNCT, CONT, PERF) é evidência de morfemas funcionais com os mesmos traços (sintáticos, semânticos e morfológicos), mas interpretados fonologicamente de maneiras distintas segundo distintas classes morfológicas que marcam formalmente os verbos (raiz + verbalizador, na ótica da Morfologia Distribuída). A homofonia de raízes nos ofereceu evidências adicionais para mantermos a nossa hipótese.

A organização em classes opera em outros domínios da língua kuikuro, além dos apresentados neste trabalho, como nas flexões dos Modos Interativos ou Performativos (Imperativo, Hortativo, Intencional) e em outros processos de nominalização. A análise das classes morfológica em Kuikuro nos coloca, contudo, uma questão sincrônica, em que processos morfofonológicos não são mais ativos. Uma futura comparação com os sistemas flexionais e derivacionais de outras línguas da família karib, com os seus ramos setentrionais e meridionais, permitirá uma visão diacrônica que poderá iluminar de outra perspectiva os fatos sincrônicos do Kuikuro.

\section{REFERÊNCIAS BIBLIOGRÁFICAS}

EMBICK, D.; HALLE, M. (2004) On the status of stems in morphological theory. University of Pennsylvania and MIT, ms.

FRANCHETTO, Bruna. (1986) Falar kuikúro. Estudo etnolingüístico de um grupo karibe do Alto Xingu. Tese de Doutorado. Rio de Janeiro, Programa de Pós Graduação em Antropologia Social, Departamento de Antropologia, Museu Nacional, UFRJ.

.(1995) Processos fonológicos em kuikúro: uma visão auto-segmental. In Leo Wetzels (org.). Estudos fonológicos das línguas indígenas brasileiras, pp. 53-84. Rio de Janeiro: Editora UFRJ.

.(2005) TAM and verbal inflection in Kuikuro (Alto Xingu, Brazil). Paper presented at Colloque sur La Grammaire des Langues Caribes, CNRS (CELIA), IRD. Paris, 5-9 de dezembro. .(2006) Are Kuikuro roots lexical categories? In Ximena Lois; Valentina Vapnarski (eds.). Lexical categories and root classes in Amerindian languages, pp. 33-68. Switzerland AG.: Peter Lang. 
FRANCHETTO, Bruna; MEIRA, S. (2005) The Southern Cariban languages and the Cariban family. International Journal of American Linguistics 17:127-190.

FRANCHETTO, Bruna; SANTOS, Mara. (2003) Natureza dos argumentos e mudança de valência a partir de uma classificação (semântica) dos "verbos" kuikuro. In F. Queixalós (org.). Ergatividade na Amazônia II, pp. 101-154. Brasília: Centre d'Études des Langues Indigènes d'Amérique (CNRS, IRD), Laboratório de Línguas Indígenas (UnB).

GILDEA, Spike. (1998) On reconstructing grammar. Comparative Cariban morphosyntax. Oxford: Oxford Universiy Press.

HALLE, M.; MARANTZ, A. (1993) Distributed morphology and the pieces of inflection. In K. Hale; S. J. Keyser (eds.). The View from Building 20. Linguistics Essays in Honor of Sylvain Bromberger, pp. 111-176. Cambridge, Mass.: MIT Press.

.(1994) Some key features of distributed morphology. In A. Carnie; H. Harley (eds). MIT Working Papers in Linguistics 21. Papers on Phonology and Morphology, pp. 275-288. Cambridge, Mass.: MITWPL.

HARLEY, H.; NOYER, R. (1998) Licencing in the non-lexicalist lexicon: nominalizations, vocabulary items and the encyclopaedia. In Heidi Harley (ed.). MITWPL 32. Papers from the Upenn, MIT Roundtable on Argument and Aspect, pp. 119-137. Cambridge: MITWPL.

SANTOS, G. M. F. dos (2002) Morfologia kuikuro: as categorias "nome" e "verbo" e os processos de transitivização e intransitivização. Dissertação de Mestrado em Lingüística. Rio de Janeiro, Universidade Federal do Rio de Janeiro, Faculdade de Letras.

.(2005a) Raízes homófonas em Kuikuro. Comunicação apresentada no XVII Congresso da ABRALIN. Brasília, 17-19 de fevereiro.

.(2005d) The inflectional morphological classes in the Kuikuro language (Alto Xingu, Brazil). Paper presented at Colloque sur La Grammaire des Langue Caribes, CNRS (CELIA), IRD. Paris, 5-9 de dezembro.

.(2007) Morfologia kuikuro: gerando nomes e verbos. Tese de Doutorado em Lingüística. Rio de Janeiro, Universidade Federal do Rio de Janeiro, Faculdade de Letras. Doutorado em Lingüística. 


\section{Abreviaturas}

$\begin{array}{ll}1 & \text { primeira pessoa } \\ 2 & \text { segunda pessoa } \\ 3 & \text { terceira pessoa } \\ \text { AN } & \text { anafórico } \\ \text { BEN } & \text { benefactivo } \\ \text { A } & \text { classe flexional }(1,2,3,4 \text { e } 5) \\ \text { CMPL } & \text { Completivo (Aspecto) }(\text { leha }) \\ \text { CONT } & \text { Continuativo (Aspecto) } \\ \text { COP } & \text { cópula } \\ \text { ERG } & \text { ergativo (heke) } \\ \text { EV } & \text { evidencial } \\ \text { ex } & \text { sufixo com significado de passado (-pe) } \\ \text { FUT } & \text { futuro } \\ \text { INST } & \text { instrumental } \\ \text { INSTNR } & \text { nominalização instrumental } \\ \text { INTR } & \text { detrasitivizador } \\ \text { LOC } & \text { locativo (em; -te) } \\ \text { MO } & \text { marcador de objeto } \\ \text { NMLZ } & \text { nominalizador } \\ \text { PERF } & \text { perfectivo } \\ \text { PL } & \text { plural } \\ \text { PNCT } & \text { Pontual (Aspecto) } \\ \text { PTP } & \text { particípio } \\ \text { REL } & \text { relacional } \\ \text { TR } & \text { transitivizador } \\ \text { VBLZ } & \text { verbalizado } \\ \text { V } & \text { verbo intransitivo } \\ \text { Vt } & \text { verbo transitivo } \\ & \end{array}$

Recebido: 20/11/2007

Versão revista: 9/11/2008

Aceito: 6/1/2009 